\title{
Performance Analysis of Acceleration Sensor for Movement Detection in Vehicle Security System
}

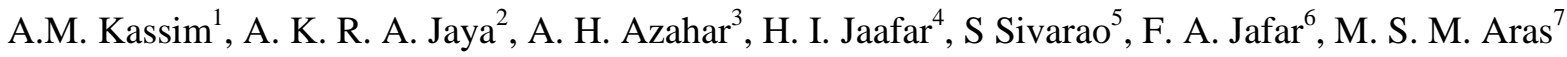 \\ Centre of Excellence for Robotic, and Industrial Automation (CERIA) \\ Universiti Teknikal Malaysia Melaka, Hang Tuah Jaya \\ Durian Tunggal, 76100 Melaka, MALAYSIA
}

\begin{abstract}
The vehicle security system is a critical part of an entire car system in order to prevent unauthorized access into the car. As the statistic has shown that the number of cases of the private car being stolen is increasing and the recovery rate is decreasing sharply, it shows that the car security system failed to perform to prevent unauthorized access. Most of the vehicle security system simply consists of a few door-open detection switches, siren, and remote control to protect the car, which appears to be weak against experienced car theft. Therefore, the project is carried out to develop a vehicle security system that can measure the dynamic acceleration inside the vehicle using the ADXL345 accelerometer and locate the coordinate of the vehicle by using U-Blox Neo-6M GPS receiver. In order to evaluate the performance of the proposed vehicle security system, the experiment to determine the most suitable position among the four places inside a car to place the device was conducted. Then, the performance analysis of the GPS receiver for accurate tracking also was done. The results showed that the most suitable position to place the device is inside the center of the car dashboard and the GPS receiver has a mean cold start-up time of 5 minutes 47 seconds and hot start-up time of 11.72 seconds, with a standard deviation of $\mathbf{0 . 0 0 0 0 0 3 7 0 6}^{\circ}$ in latitude and $0.000002762^{\circ}$ in longitude for position tracking.
\end{abstract}

Keywords-Security system; acceleration sensor; movement detection; ADXL345

\section{INTRODUCTION}

The vehicle security system is a critical part of an entire car system in order to prevent unauthorized access into the car. Unfortunately, most of the car security system failed to prevent car theft. Starting from the first case of car theft in the year 1896 , the number of cars being stolen has been raised rapidly. The statistic of the number of stolen private cars in Malaysia is increased drastically from 1980 to 2010 Although there is some decrease in the stolen cases for some years the number of cases is raised rapidly overall. The statistic shown that the number of cases of the private car being stolen is increasing year by year, it shows that the car security system failed to perform to prevent unauthorized access[1-2].

In addition, there are many methods used by car theft. The study conducted in 2009 in the United Kingdom, most cases happen when the car keys are being stolen in the burglary, which is $37 \%$ [2]. Followed by the cases where the owner left their car key inside the car (18\%). 14\% of the car stolen by forced ignition, where the theft hijacks the car wiring system to start the car and drive away. Hence, the conclusion that $72 \%$ of the cases are related to car keys (Other using keys 12\%, Keys stolen in burglary $37 \%$, Keys stolen in robbery $5 \%$, Keys left in car 18\%) which reflects that car keys failed to protect the $\operatorname{car}[3-5]$.

On the other hand, there is some lucky owner who could recover back their car after being stolen by car theft. The recovery rate for the year 2003-2004 (59.4\% total) is higher than 2008-2009 which is only $10 \%$ of total cases. The recovery rate dropped sharply due to the rapid rise in the number of cases of a stolen vehicle in 2008-2009. With the low recovery rate, the performance and effectiveness of the vehicle security system should be taken into consideration seriously to prevent further increase in vehicle stolen cases [1]. Most of the cars today are equipped with a security system as a vehicle security system is becoming "must-have" equipment for the safety of every car. Different types of security systems are designed by a manufacturer or third party organization according to the car owner's need, some may include a vibration sensor or even a tilt sensor for additional protection.

Some researchers presented the vehicle security system, which consists of the remote keyless entry (RKE) and Immobilization with the use of the microcontroller unit (MCU). This system improves the safety of remote key control by introducing a unique authentication technique in which the remote unit generates different authentication codes to the MCU during each transmission which makes the decoding job difficult for car theft. This immobilizer system works by implementing an Integrated Circuit (IC) inside the car key remote control unit which communicates with the MCU by using a low frequency (LF) radio signal at $125 \mathrm{kHz}$ with inductive coupling technique. After receiving the correct authentication code, the MCU will allow the engine to be started up by the user. This system has the advantages of secure communication which makes the radio intercept and replay technique failed to gain access to the vehicle[6-10].

In addition, there are some researchers also come out with a vehicle security system using radio-frequency identification (RFID). This system reduced the hacking probability to $10^{-14}$ by sending an 8-bit rolling unique authentication code each time the transmitter operates. Compared to passive RFID, active RFID has a longer range of up to $200 \mathrm{~m}$ for operation, which enables the owner to disable the stolen car in a safe distance. This system consists of three units which are transmitting unit, receiving unit and intelligent vehicle control unit that communicating with each other. In case a vehicle is in danger, the owner can simply press the disable button on the portable transmitting unit, which will then sends out the unique 
authentication code to the receiving unit. After the receiving unit decoded the signal, the receiving unit communicates with the intelligent vehicle control unit to turn off the car engine. One of the advantages of this system is that the system includes an automatic gear shifter controller, which will shift the transmission to the neutral position in case the vehicle is at high speed and allows the vehicle to bring down the speed before the engine shuts down [11-14].

Iman M.Almomani, et al. and some researchers also designed a global positioning system (GPS) vehicle tracking and management system. This system combined both the global system for mobile communication (GSM), general packet radio service (GPRS) and GPS to achieve vehicle tracking. The GSM is used to send an alert message through short messaging service (SMS) which consists of four types of alert: over-speeding, enter/ exit a geofence area, car stops/ moves, and alarm status. GPRS is used to upload the tracking data onto GPRS Server in the user-defined interval which then allows online tracking via Web Server. Google Maps is used to display the vehicle position, which includes the geometrical information and graphical presentation, allows the user to recognize the vehicle's position easily. This system has the advantages of being user-friendly and easy management via the use of a computer or mobile phone. On the other hand, this system has disadvantages require internet connectivity to be functional [15-17].

Moreover, most of the vehicle security system simply consists of a few door-open detection switches, siren, and remote control to protect the car from unauthorized access, which appears to be weak against an experienced car thief. Besides, a remote control based vehicle security system only allows one way of communication, remote-to-car, this limitation causing the remote control based vehicle security system becoming passive as the owner only able to know the car is being intruded when he/she hears the siren. If the owner is inside a building, the system fails to inform the owner that his/her car is in danger. Besides, the conventional vehicle security system is not able to detect the location and movement of the vehicle, which fails to protect the car if it's pushed or towed away [18-20].

Hence, these issues lead to the development of a more advanced vehicle security system which is able to detect movement and/or vibration of the vehicle with location coordination reporting and two-way communication between the owner and the security system. The appropriate sensor will be selected to trigger the security system and immobilizer will be added to turn off the car engine after the movement has been detected.

\section{HARDWARE CONFIGURATION}

\section{A. System Overview}

The system overview is illustrated in Fig. 1 shows the relationship for each component to the microcontroller. The development of prototype consists of two parts where the first part is the hardware selection and construction. The hardware consists of a sensor, microcontroller, and actuator. The second part is about the development of software, the software is the coding programmed into the microcontroller for the control part. Microcontroller a device made up of processor, RAM, ROM, input and output (I/O) peripherals into an integrated circuit. Arduino microcontroller is preferred as it is opensource and it provides free content to be downloaded from the internet. Arduino Pro Mini 328 is chosen for this project as it has a small form factor of $18 \mathrm{~mm}$ x $33 \mathrm{~mm}$, which is ideal for a vehicle security system where hidden installation can be made easily.

The Arduino microcontroller running at a clock speed of 16 $\mathrm{MHz}$ and provides 14 digital input/output (I/O) ports and eight analog input which is exactly the same as the famous Arduino Uno R3 microcontroller. Although it is small, it provides the serial peripheral interface (SPI) and I2C protocol for connecting sensors. In this project, Arduino Pro Mini 328 that is shown in Fig. 2 is used to process sensor reading from the ADXL345 accelerometer, U-Blox Neo-6M GPS receiver, RF remote module and GSM modem by C-language programming using Arduino Integrated Development Environment (IDE) software.

The ADXL345 accelerometer sensor comes with a small and thin integrated circuit and the module used in this project is made by the CJMCU Company. This module features dual voltage supply options of $5 \mathrm{~V}$ and $3.3 \mathrm{~V}$ for user convenient. ADXL345 accelerometer sensor is able to measure acceleration in $\mathrm{X}, \mathrm{Y}$ and $\mathrm{Z}$-axis with high resolution of 13-bits and userconfigurable measurement range of $\pm 2 \mathrm{~g}, \pm 4 \mathrm{~g}, \pm 8 \mathrm{~g}$ and $\pm 16 \mathrm{~g}$, which is more than enough for this project. Besides, the ADXL345 also features SPI (3 or 4 wire) and I2C digital interface for interfacing with microcontroller. The ADXL345 provides the measurement of tilt angle by measuring the static acceleration of gravity and dynamic acceleration in 3-axis, which is suitable for this project to detect any vehicle movement in all axis and transmit to the microcontroller. Fig. 3 shows the ADXL345 accelerometer used in this project.

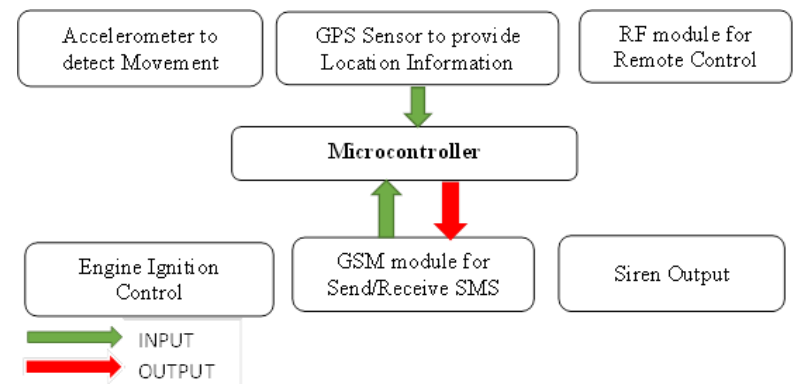

Fig. 1. System overview of Acceleration-based Movement Detection.

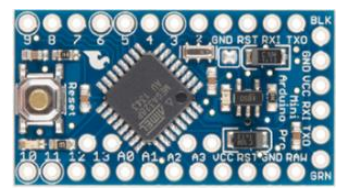

Fig. 2. Arduino Pro Mini 328.

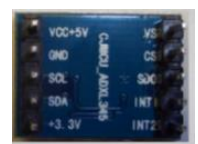

Fig. 3. CJMCU_ADXL345 Accelerometer Sensor. 
On the other hand, the global positioning system (GPS) receiver is a device which gathers satellite signal from the GPS satellite and calculates the position based on the received signal. The U-Blox Neo-6M GPS device, which is shown in Fig. 4, have featured high-performance tracking ability while maintaining cost-effective which makes it suitable for this project. This sensor can be connected by UART, USB, SPI and I2C protocol which makes it popular across many platforms. The cold start of the sensor takes 27 seconds for the first reliable reading at outdoor and a 1 second for hot-start ability. The U-Blox Neo 6M has a horizontal accuracy of $2.5 \mathrm{~m}$ which is enough for the tracking purpose. In addition, this sensor also provides velocity output at an accuracy of $0.1 \mathrm{~m} / \mathrm{s}$ and an altitude reading of a maximum of $50,000 \mathrm{~m}$. In this project, the U-Blox Neo-6M GPS sensor will be connected to the Arduino Pro Mini microcontroller via UART protocol at 9600 baud rate and a reading update rate of $1 \mathrm{~Hz}$.

In addition, the GSM modem is a module that enables communication, SMS and internet connectivity over the cellular network. The Neoway M590E operates on the GSM network at $900 \mathrm{MHz}$ and $1800 \mathrm{MHz}$ which is supported in most countries and generally classified as $2 \mathrm{G}$ Network. A Sim card is needed for proper identification at Telecommunication Company. This module comes in the small form factor of 27.6 $\mathrm{mm} \times 21.2 \mathrm{~mm}$ which enables a compact arrangement of this project. The Neoway M590E enables communication between the user and the Arduino Pro Mini by Send and Receive SMS. It connects with the microcontroller by using the UART protocol at 4800 baud rates. Fig. 5 shows the hardware overview of system configuration for each component used in this proposed system.

\section{B. Acceleration Measurement Method}

Fig. 6 shows the flowchart of the acceleration measurement part when the alarm is turned $\mathrm{ON}$ by the user, the sensor will be initialized with the first measurement reading as a reference point. After initializing, the sensor will measure the dynamic acceleration continuously to detect changes in acceleration in $\mathrm{X}, \mathrm{Y}$ and $\mathrm{Z}$-axis. If a measured acceleration value is higher than the threshold value, the siren alarm is triggered and followed by the activation of the engine ignition control relay, which will turn of the power of the ignition system. SMS will be sent to alert the user.

The output of the ADXL345 sensor through the I2C protocol needs to be filtered by taking average value to minimize the effect of noise. This is achieved by taking an average of five readings continuously and calculate the average value in 3-axis. The value is then converted to unit $\mathrm{G}$ based on the formula stated in ADXL345 datasheet as shown below. $( \pm 2 \mathrm{~g}$ measurement range)

$X_{g}=x\left(\frac{4}{1024}\right)$

$Y_{g}=y\left(\frac{4}{1024}\right)$

$Z_{g}=z\left(\frac{4}{1024}\right)$

\section{GPS Localization}

Fig. 7 shows the flowchart of the GPS locating part of the project. From the datasheet, the U-Blox Neo-6M GPS Receiver will receive the GPS satellite signal and output national marine electronics association (NMEA) GPS data to the Arduino Pro Mini microcontroller. The microcontroller will decode the NMEA data by using the TinyGPS library [21] into GPS coordinate in Latitude and Longitude. These values will be stored in the microcontroller RAM as the Last Known Position. A link to Google Maps will be generated by using the Last Known Position and sent to the user when requested or intrusion detected. Formula (4) below shows the method of generating a Google Map Link. [22]

http://maps.google.com/?q=<lat $>,<\operatorname{lng}>$

where <lat $>$ is the Latitude and $<\operatorname{lng}>$ is the Longitude.

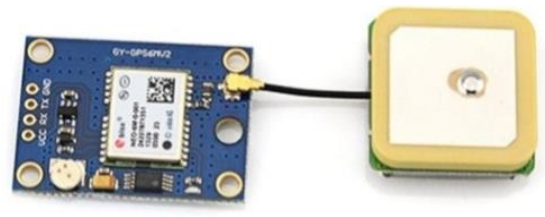

Fig. 4. U-Blox Neo-6M GPS Sensor.

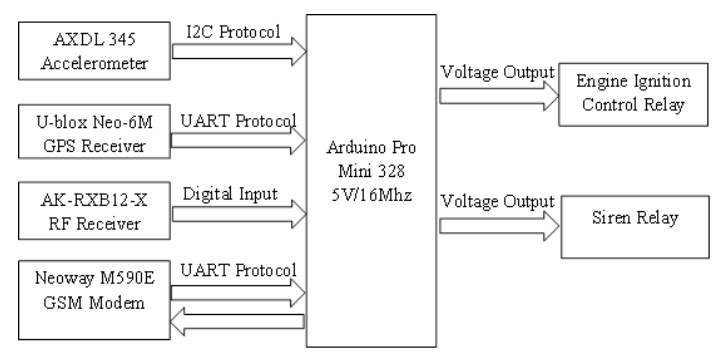

Fig. 5. Hardware Overview.

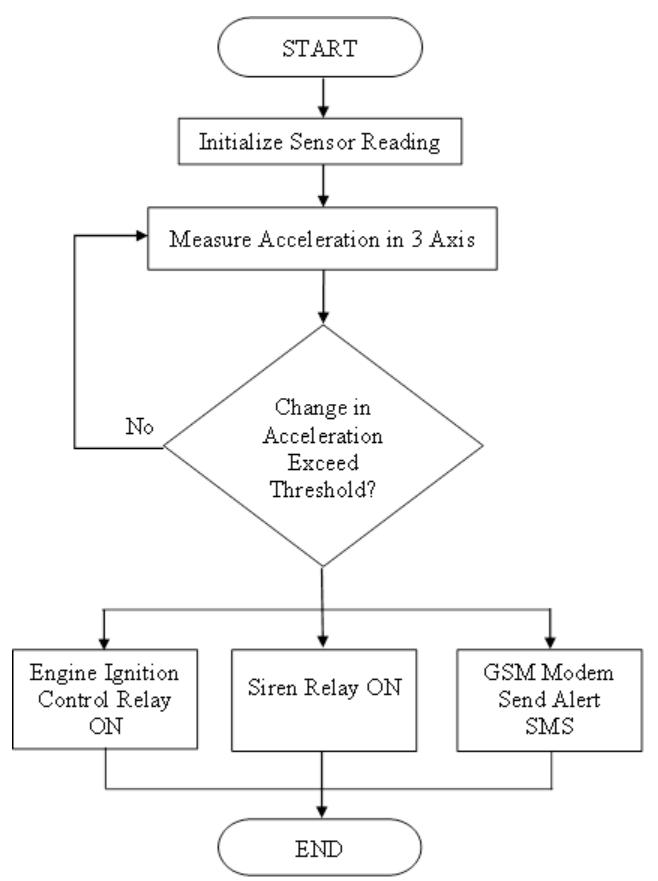

Fig. 6. Flowchart of the Acceleration Measurement. 


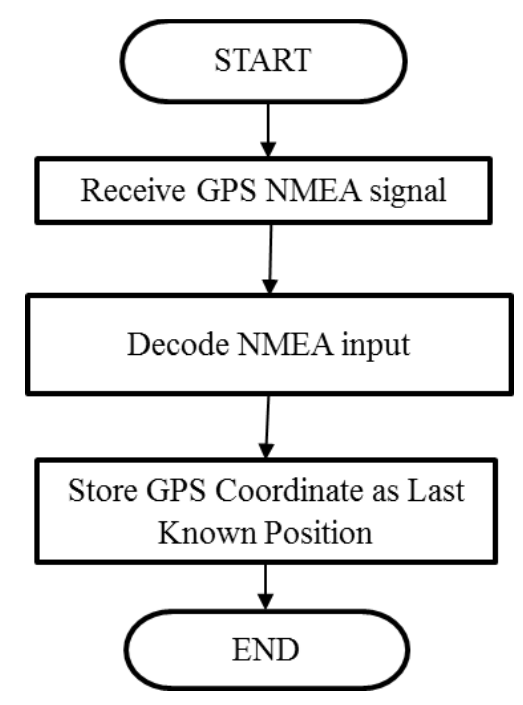

Fig. 7. Flowchart of the GPS Localization.

\section{EXPERIMENTAL SETUP}

\section{A. Experiment 1: Performance Analysis for Device Installation Place}

In experiment 1 , the objectives are to compare and find the best position of the accelerometer inside a car which gives a better reading when starting the engine. Measurement was made in four positions inside a car: 1) Center of the dashboard, 2) Storage compartment, 3) Under driver seat, and 4) Back bonnet. A prototype consists of accelerometer ADXL345, Arduino Pro Mini 328 and a Bluetooth module is developed to carry out this task. The Bluetooth adapter is used to transfer the measured acceleration data to the laptop wirelessly, which can reduce the error in measurement caused by the data cable. Fig. 8 shows the set-up of the prototype used in this experiment.

In order to maximize the validity of data measured from the experiment, three variable is identified before the experiment, which is an acceleration in X-Axis, Y-Axis, and Z-axis in-unit $\mathrm{g}$ (gravitational acceleration which is equal to $9.81 \mathrm{~m} / \mathrm{s}$ ). All the measurement is made in the same car, which is Proton Exora 1.6 CPS MT in this experiment, to increase the reliability of the measured data. The prototype is also secured to the surface using double-sided tape to ensure no sliding occurs. Besides, the orientation of the device is also fixed so that the three-axis did not interchange for better comparison.

\section{B. Experiment 2: Performance Analysis for the GPS Receiver for Accurate Tracking}

In experiment 2 , the objective is to locate the coordinates of the device in a fixed position. The data from the U-Blox Neo6M GPS Receiver will be collected and analyzed, which includes cold-start time (time from power on until first valid reading without satellite data in memory), hot-start time (Time from power on until valid reading with satellite data in memory), mean coordinate and standard deviation. By collecting this data, the performance of the device to locate the coordinate can be analyzed. Fig. 9 shows the working principle of the coordinate locating prototype.

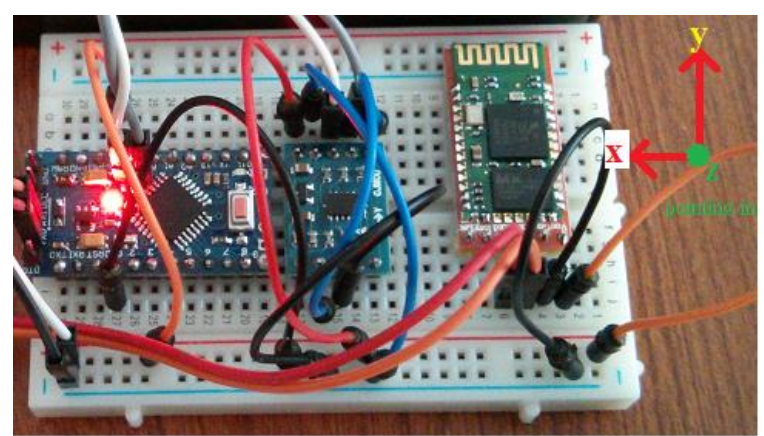

Fig. 8. The Orientation of the Acceleration Measurement Prototype Across all Measurement.

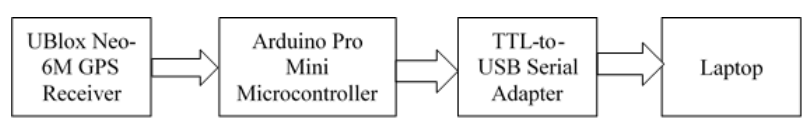

Fig. 9. Working Principle of the Coordinate Locating Prototype.

The cold-start time is measured by taking the time taken from power until the first valid reading shown in the serial monitor on the laptop. This process is repeated five times to get the mean cold-start time. The hot-start time is measured by taking the time taken from power loss of the device to resumed reading after power on in serial monitor on the laptop. This process also repeated five times to get a mean value. The mean coordinate and standard deviation is calculated from 20 times measurement at a fixed position.

\section{EXPERIMENTAL RESULTS}

\section{A. Performance Analysis for Device Installation Place}

Fig. 10 to 13 show the acceleration measured at a different position from experiment 1 such as at the center of the dashboard, storage compartment, under the driver seat, and back bonnet. The Z-axis experienced a steady $1 \mathrm{~g}$ of acceleration from the gravity acting towards the ADXL345 sensor. It was found that the Y-axis shows a little bit of deviation from the 0 point, this may due to the calibration error inside the sensor. From all the figures, the Z-axis shows the most changes in acceleration across all positions compared to the $\mathrm{X}$-axis and $\mathrm{Y}$-axis, which is doesn't react much to the starting of the car engine.

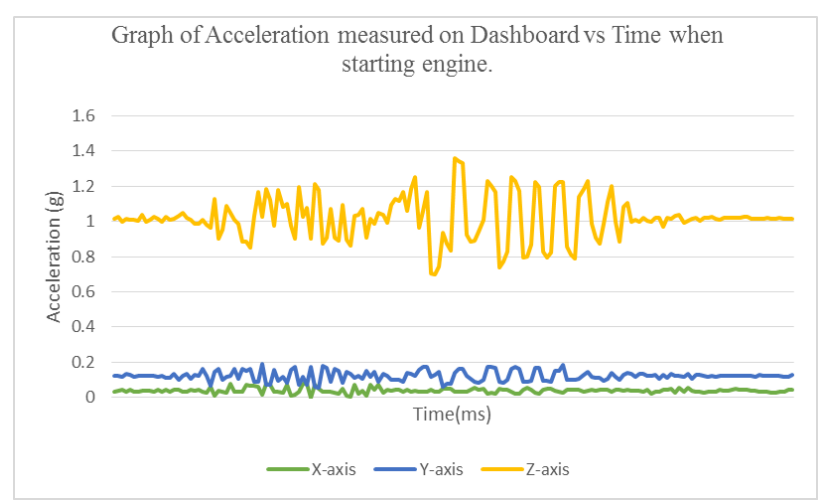

Fig. 10. Installation at the Center of Dashboard Result. 


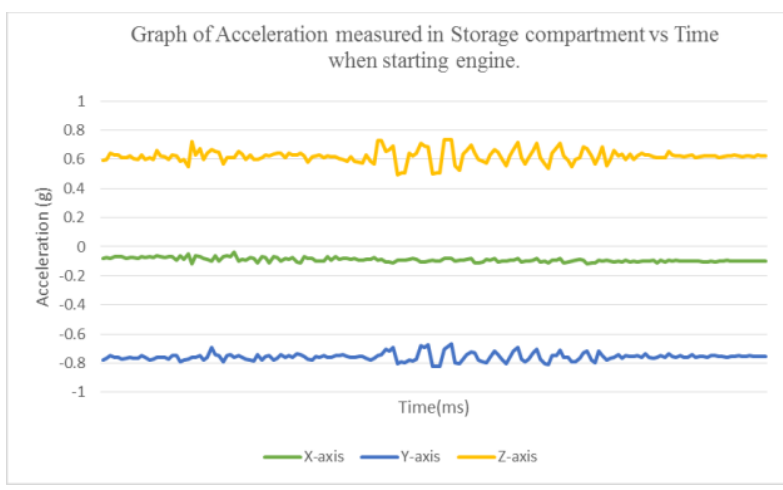

Fig. 11. Storage Compartment Result.

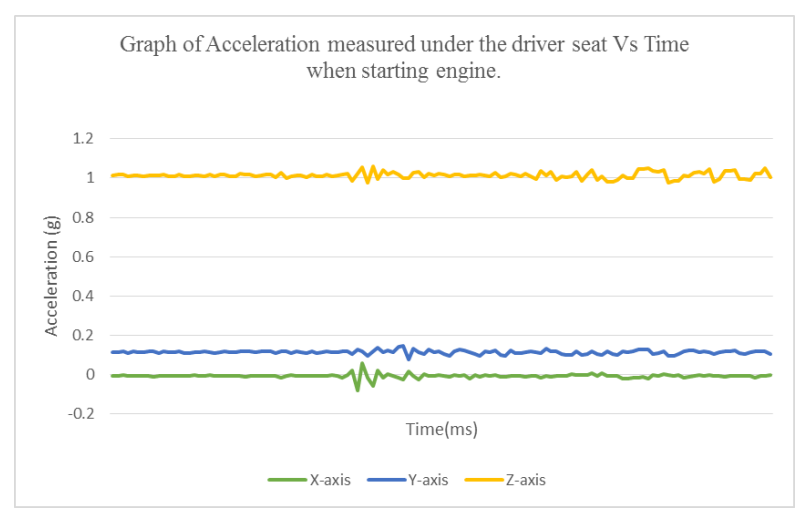

Fig. 12. Under Driver Seat Result.

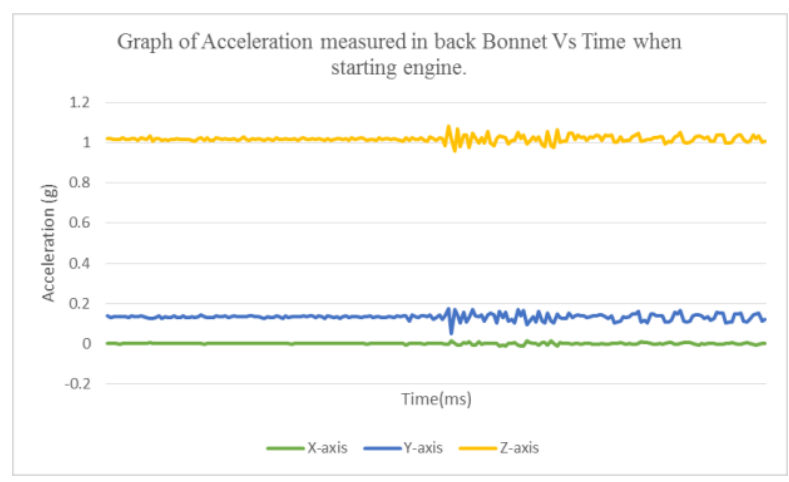

Fig. 13. Back Bonnet Result.

The vibration of the car engine causes this condition during starting, as the engine of the Proton CPS engine used in the testing car is an inline engine, the piston moves upward and downward for each burning cycle, which causes the vibration at the $\mathrm{Z}$-axis. For the $\mathrm{X}$-axis and $\mathrm{Y}$-axis, they do not experience huge changes in acceleration value due to the car is stationary during testing. Fig. 14 shows the comparison of the Z-axis for the four positions together. From the graph, it was found that the Z-axis acceleration value has deviated from the rest, which is caused by the Storage compartment has an angled design at the bottom and this makes the prototype hard to achieve the standard measurement position.

The center of the dashboard position shows the highest change in acceleration at Z-axis among all the positions, which makes it the best position to install this project compared to the others. Under-seat and bonnet position shows the lowest change in acceleration compared to the others, which is not suitable for the installation of this project. This condition can be explained by the distance from the engine to the device. As the testing car has an FF (front-engine front-wheel-drive) configuration, the engine is located at the front of the car. This explains the large changes of acceleration detected on the dashboard and storage compartment due to the short distance to the car engine. On the other hand, the bonnet and under-seat position are far away from the car engine which results in lower amplitude readings. In order to improve the security of the device while maintaining high sensitivity, the device will be installed inside the dashboard which makes the car theft harder to access. The device is simply placed on top of the dashboard due to the difficulty to remove the dashboard on the testing car that is too high.

\section{B. Performance Analysis for of the GPS Receiver for Accurate Tracking}

Fig. 15 shows the time needed from the power-on of the GPS module until the first valid reading is getting from the GPS module (time-to-first fix). Cold-start of the GPS module is carried out when the GPS module is powered off without backup supply for more than 4 hours. The data collected in Fig. 15 shows that the U-Blox Neo-6M GPS module's coldstart time is between 291 seconds to 417 seconds with a mean of 347 seconds and a standard deviation of 48.929 seconds.

Fig. 16 shows the time taken for the hot-start action of the GPS module. The hot-start action of the GPS Module is carried out when the GPS module is powered on from the state of standby with all the previous satellite locating data inside the memory. The hot-start action can be carried out even the module is powered off when there is a backup power to support the memory chips on the module. From Fig. 16, the hot-start time varies from 9.90 seconds to 15.21 seconds with a mean of 11.72 seconds and a standard deviation of 2.162 seconds.

Fig. 17 and 18 show the 20 sets of GPS coordinate data collected from experiment 2 and converted into a graph for better analysis. From the graph, the GPS coordinates from the U-Blox Neo-6M GPS module can be said consistence, with a mean GPS coordinates of latitude $2.25102195^{\circ}$ and longitude $102.2859226^{\circ}$. The standard deviation for latitude and longitude is $0.000003706^{\circ}$ and $0.000002762^{\circ}$, respectively. This result shows that the GPS coordinates collected from the GPS module at a fixed position have a high precision which is suitable for tracking purposes for this project.

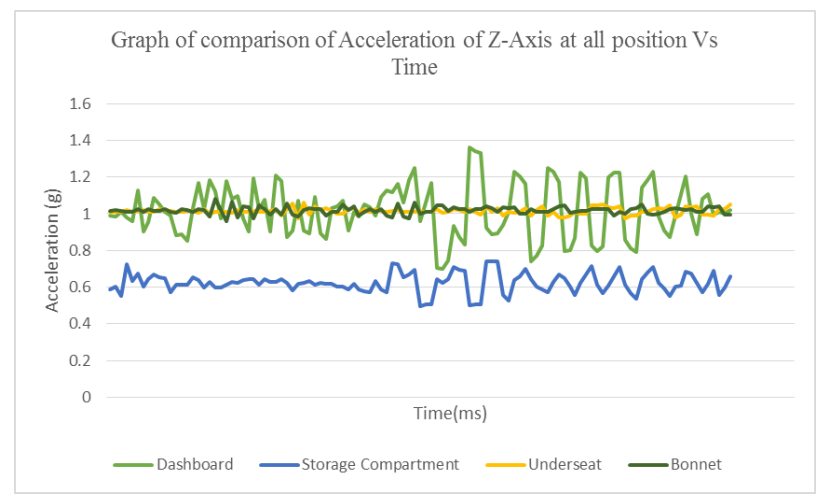

Fig. 14. Comparison of Z-Axis at all Position. 


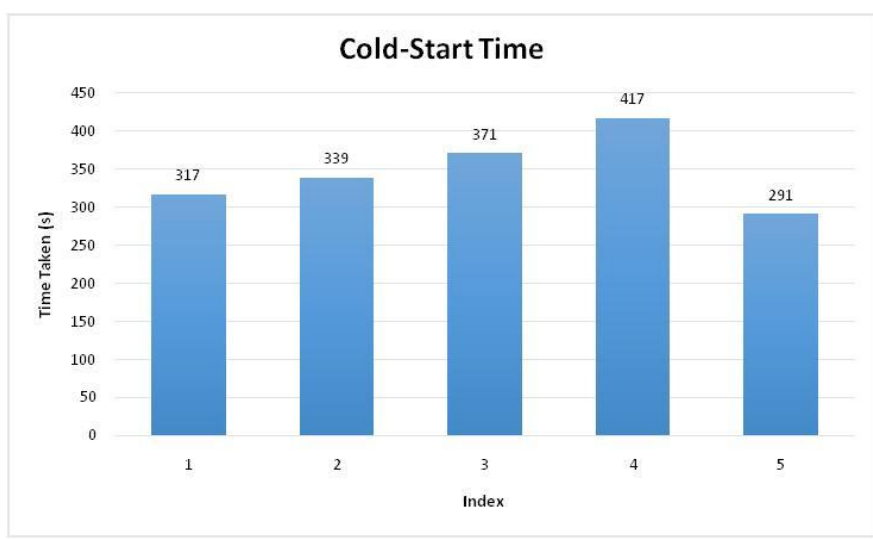

Fig. 15. Time is Taken for Cold Start-ups.

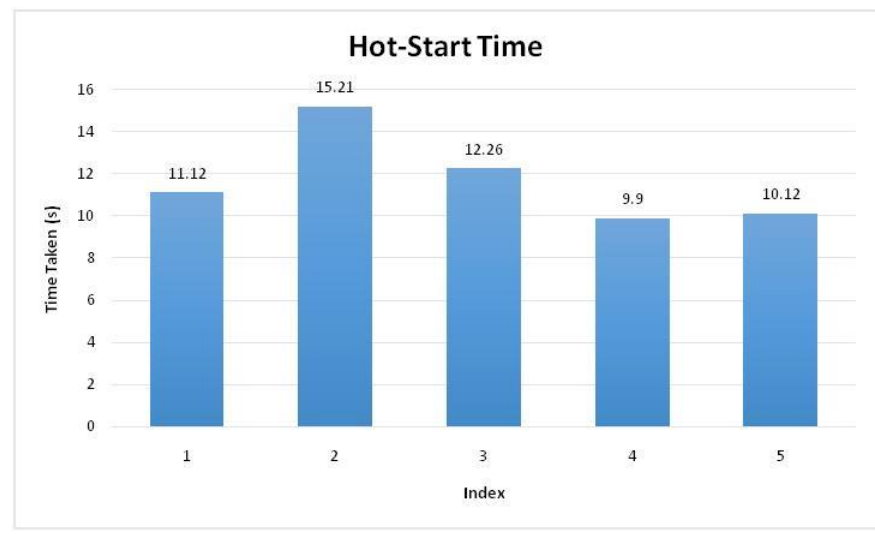

Fig. 16. The time is taken for a hot start-up

Based on these results, the accuracy of the GPS coordinates to be detected during the cold-start and hot-start time are evaluated. The cold-start time and hot-start time collected in experiment 2 are important in determining the performance of the GPS module. A shorter time to first valid fix is preferred so that the user able to locate the vehicle faster. Besides, the hotstart action is preferred over the long cold-start action, hence, the GPS module should be always powered on and going to standby mode if power saving is required.

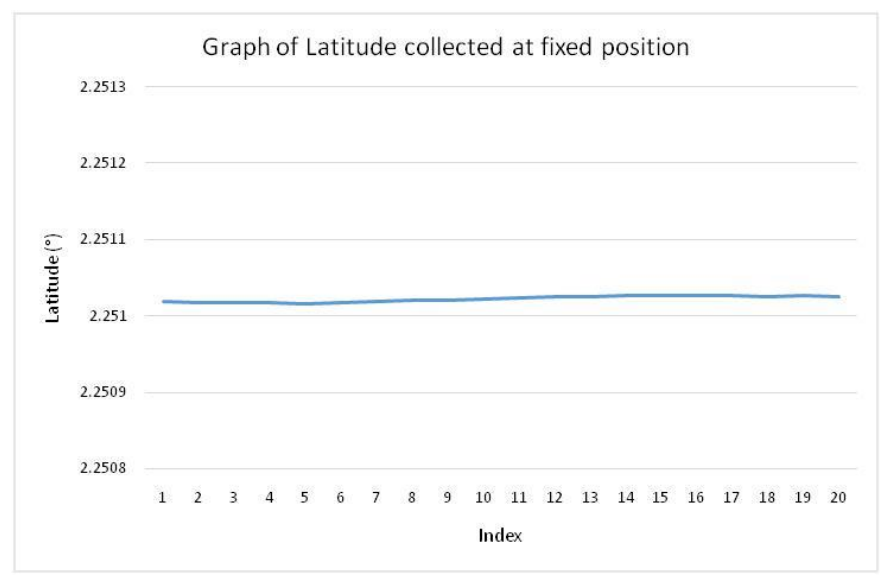

Fig. 17. The Graph of Latitude Collected from GPS at a Fixed Position.

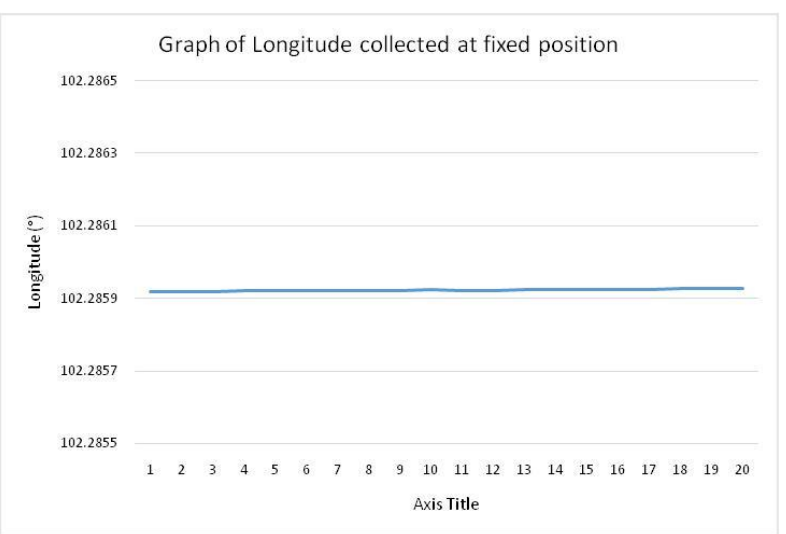

Fig. 18. The Graph of Longitude Collected from GPS at a Fixed Position.

\section{CONCLUSIONS AND Future TASKS}

In conclusion, the first objective which is to measure dynamic acceleration with accelerometer ADXL345 in X-axis, Y-axis, and Z-axis for movement and vibration detection, based on installation location is achieved in the experiment 1 . The analysis of the data taken from the experiment is useful in determining the installation position of the device and method of detecting the intrusion of car theft. The center of the dashboard is the best position to install the vehicle security system and the best detecting method by measuring the vibration of the car engine in the Z-axis. In experiment 2 , the performance of the GPS module is evaluated for use in this project. The GPS coordinates collected from the GPS module at a fixed position has a high precision which is suitable for tracking purpose for this project.

For future development of the vehicle security system, door open detection switch can be included to further improve the ability to detect intrusion of car theft. The door open detection switch plus the acceleration detection can work together to detect intrusion at a different level of alert. Besides, the backup battery can be included in this project to provide an emergency power source in case the wiring system has been modified so that the user is still able to know the coordination of the vehicle by SMS.

\section{REFERENCES}

[1] C.Teh, "Private car theft statistics in Malaysia: One Stolen every 24 minutes." Available at: http://christopherteh.com/blog/2010/09/ privatecar-theft-statistics-in-Malaysia-one-stolen-every-24-minutes/ (2019).

[2] Rosenfeld, R. "Crime and Inflation in Cross-National Perspective". Crime and Justice, 43(1), 341-366, 2014.

[3] N. Morgan, O. Shaw, A. Feist, and C. Byron, "Reducing criminal opportunity: vehicle security and vehicle crime", Research Report 87, January 2016.

[4] Kamkar, S. "Drive It Like You Hacked It: New Attacks and Tools to Wirelessly Steal Cars". Presentation at DEFCON 23, August 2015.

[5] Brown, R., Explaining the property crime drop: The offender perspective. Trends \& issues in crime and criminal justice". No. 495. Australian Institute of Criminology, 2015.

[6] B. Davis, R. DeLong, "Combined Remote Key Control and Immobilization System for Vehicle Security", Motorola Semiconductor Products Sector, Arizona, pp 125- 132, 1996.

[7] Van Ours, J. C. \& Vollaard, B., "The Engine Immobiliser: A NonStarter For Car Thieves". The Economic Journal, 2014. 
[8] Brown, R., "Reviewing the effectiveness of electronic vehicle immobilization: Evidence from four countries". Security Journal, 2013.

[9] A.M Kassim, M.S Jamri, M.S.M Aras, M.Z.A Rashid, "Design and Development of Vibration Method for Vehicle Reverse System (VRS)," Procedia Engineering, Vol. 41 pp. 1114-1120, 2012.

[10] F. D. Garcia, D. Oswald, T. Kasper, P. Pavlidès, "Lock it and still lose it-on the (in) security of automotive remote keyless entry systems", Proc. 25th USENIX Security Symp. (USENIX Security), pp. 929-944, 2016.

[11] G. Jayendra, S. Kumarawadu, L. Meegahapola, "RFID-Based Anti-theft Auto Security System with an Immobilizer", University of Moratuwa, Sri Lanka, pp 441-446, 2007.

[12] Kasper, T., Oswald, D., and Paar, C. "Wireless security threats: Eavesdropping and detecting of active RFIDs and remote controls in the wild". In 19th International Conference on Software, Telecommunications and Computer Networks - SoftCOM'11 pp. 1-6, 2011.

[13] A. Kassim, H. Jaafar, M. Azam, N. Abas, and T. Yasuno, "Design and development of navigation system by using RFID technology," in Proc. IEEE 3rd Int. Conf. Syst. Eng. Technol. (ICSET), pp. 258-262, Aug. 2013.

[14] Tseloni, A., Thompson, R., Grove, L., Tilley, N. \& Farrell, G, "The effectiveness of burglary security devices. Security Journal". Available at: http://www.palgrave-journals.com/sj/journal/vaop/ncurrent/pdf/ sj201430a.pdf, 2014.
[15] A. M. Kassim, T. Yasuno, H. Suzuki, H. I. Jaafar, M. S. M. Aras, "Indoor navigation system based on passive RFID transponder with digital compass for visually impaired people", Int. J. Adv. Comput. Sci. Appl., vol. 7, no. 2, 2016.

[16] K. T. Cho, K. G. Shin, "Fingerprinting electronic control units for vehicle intrusion detection", Proc. 25th USENIX Security Symp., pp. 911-927, 2016.

[17] I. M. Almomani, N. Y. Alkhalil, E. M. Ahmad and R. M. Jode, "Ubiquitous GPS Vehicle Tracking and Management System", University of Jordan, Jordan, 2011.

[18] Choudhuri, S. B., Kumar, J. S. J., Venkatesh, B. \& Pandey, R. K., "Vehicle Anti-Theft and Passenger Safety System". Global Journal of Research and Engineering-GJRE-B, 14(1), 2014.

[19] M. Z. M. Rashid, H. N, M Shah, M. S. M Aras, M. Kamaruddin, A. M. Kassim, H. I. Jaafar, "Metal Line Detection: A New Sensory System For Line Following Mobile Robot", Journal of Theoretical and Applied Information Technology, vol. 64, no. 3, pp. 756-764, June 2014.

[20] A.M. Kassim, H. I Jaafar, M.A. Azam, N. Abas, T.Yasuno, “ Performances study of distance measurement sensor with different object materials and properties" 3rd IEEE International Conference on System Engineering and Technology (ICSET), pp. 281-284, 2013.

[21] M. Hart, "TinyGPS Library". Available at: http://arduiniana.org/ libraries/tinygps/ (2019).

[22] R. Rodger, "Beginning Mobile Application Development in the Cloud", Crosspoint Boulevard, Indianapolis, 2012. 